\title{
Prolonged Activated Clotting Time Immediately Prior to Open Cardiac Surgery
}

\author{
Sarah M. Baker, MSc, ${ }^{1}$ Ashley Kiefer, MD, ${ }^{2}$ Dominic S. Carollo, MD, ${ }^{3,4}$ Rajasekharan P. Warrier, MD ${ }^{4,5}$ \\ 1Department of Microbiology and Immunology, Tulane University School of Medicine, New Orleans, LA ²e Bonheur Children's Hospital, \\ University of Tennessee, Memphis, TN ${ }^{3}$ Department of Anesthesiology, Ochsner Clinic Foundation, New Orleans, LA ${ }^{4}$ The University \\ of Queensland School of Medicine, Ochsner Clinical School, New Orleans, LA ${ }^{5}$ Department of Pediatrics, Ochsner Clinic Foundation, \\ New Orleans, LA
}

Background: Prekallikrein deficiency is an extremely rare disorder in which functional prekallikrein in the plasma is reduced or absent. Case Report: We present the case of a 15-year-old male with prolonged activated clotting time incidentally noted preoperatively prior to repair of an atrial septal defect. The patient was subsequently found to have prekallikrein (Fletcher factor) deficiency. He successfully underwent open cardiac surgical repair after fresh frozen plasma was administered at a dose of $15 \mathrm{~mL} / \mathrm{kg} 1$ hour prior to the start of the procedure.

Conclusion: History and routine preoperative evaluations of complete blood count, partial thromboplastin time, prothrombin time, and platelet function analysis failed to detect any abnormalities, but a prolongation of activated clotting time identified by the anesthesiologist led to specialized testing and a diagnosis of Fletcher factor deficiency. The patient tolerated the open-heart surgery well without any significant hematologic intervention or complications.

Keywords: Factor XIla, prekallikrein, prekallikrein deficiency

Address correspondence to Rajasekharan P. Warrier, MD, Department of Pediatrics, Ochsner Clinic Foundation, 1315 Jefferson Hwy., New Orleans, LA 70121. Tel: (504) 842-4540. Email: rwarrier@ochsner.org

\section{INTRODUCTION}

Prekallikrein deficiency, an autosomal recessive disease first described in 1965 by Hathaway and colleagues, ${ }^{1}$ is an extremely rare disorder in which functional prekallikrein in the plasma is reduced or absent. Fewer than 80 cases have been reported in the literature. ${ }^{2}$ We report the case of a patient who was found to have prekallikrein deficiency after a perioperative finding of significantly prolonged activated clotting time $(A C T)$.

\section{CASE REPORT}

A 15-year-old African American male football player with a history of atrial septal defect (ASD) not amenable to device closure was noted to have a significantly prolonged ACT of 424 seconds (4 times normal) after the placement of arterial lines and after induction of anesthesia, just prior to the start of surgical closure of his ASD. Surgery was immediately halted, and an emergency consult was placed to pediatric hematology. The patient reported no significant bleeding history, including no history of bleeding from circumcision, injuries during football, or dental extraction. Family history was also negative for known bleeding disorders. His physical examination was significant only for a grade II/VI systolic ejection murmur heard best along the left upper sternal border, secondary to his ASD.
Diagnostic evaluation revealed persistently elevated partial thromboplastin time (PTT) (Table). Prothrombin time (PT); international normalized ratio; factor XIII, IX, and XI; Fitzgerald factor; fibrinogen; and liver function tests, drawn at subsequent hematology visits, were within normal range. The PTT failed to correct on a 1:1 mix and incubation, initially suggesting the presence of an inhibitor. Lupus anticoagulant was transiently elevated, with resolution on repeat testing, and repeat mixing studies were inconsistent. The diagnostic evaluation prompted continued analysis that revealed low plasma prekallikrein activity and elevated high molecular weight kininogen (HMWK) activity (Table). These findings confirmed the diagnosis of prekallikrein (Fletcher factor) deficiency. The patient was cleared for surgery with the recommendation that fresh frozen plasma (FFP) be administered at a dose of $15 \mathrm{~mL} / \mathrm{kg} 1$ hour prior to the start of the procedure. The administration of FFP allowed for normalization of prekallikrein and thus improved monitoring during surgery. The open cardiac surgical repair was performed at another institution without complications, and the patient recovered well. Genetic testing was recommended for family members.

\section{DISCUSSION}

Prekallikrein is a glycoprotein synthesized in the liver. More than $75 \%$ of prekallikrein circulates as a complex with 
Table. Results of Patient's Diagnostic Evaluation

\begin{tabular}{lcc}
\hline Variable & Results & $\begin{array}{c}\text { Reference } \\
\text { Range }\end{array}$ \\
\hline Activated clotting time, seconds & 424 & $70-150$ \\
Partial thromboplastin time, seconds & 37.8 & $21.0-32.0$ \\
& 50.2 & \\
& 35.6 & \\
Prekallikrein activity, \% & $<5$ & $63-135$ \\
High molecular weight & 153 & $65-135$ \\
kininogen activity, \% & & \\
\hline
\end{tabular}

Note: Prolonged activated clotting time was noted immediately prior to the initial planned surgical procedure. Partial thromboplastin time, prekallikrein activity, and high molecular weight kininogen activity were determined at subsequent hematology visits.

HMWK, and together with factor XII and HMWK, constitutes the contact phase of the intrinsic coagulation and fibrinolytic pathways. $^{3}$ This disorder is unique in that the coagulation pathology presents with in vitro clotting defects, usually without in vivo clotting disorders. Consequently, the deficiency is asymptomatic, with little risk of bleeding. ${ }^{4}$ However, rare cases are associated with recurrent mucosal bleeding, ${ }^{5}$ hypertension, ${ }^{6}$ recurrent cerebrovascular accidents, ${ }^{7}$ recurrent pregnancy loss, ${ }^{8}$ or failure to progress during labor. ${ }^{9}$ Interference with the in vitro measurement of ACT and PTT could cause significant difficulties during the perioperative and postoperative monitoring of anticoagulation that is routinely done with cardiac surgery. The potential for interference with the measurement of ACT and PTT emphasizes the importance of physician understanding of the limitations of laboratory testing. The ACT assay requires endogenous kallikrein-kinin system factors for activation of the coagulation pathway in vivo. Therefore, others have recommended the use of the plasma anti-Xa heparin activity assay to monitor anticoagulation in patients with prekallikrein deficiency. ${ }^{10}$

A differential for a normal PT with a prolonged PTT should include disorders of the intrinsic pathway of coagulation. These disorders include inherited deficiencies of factors VII, IX, and XI that present with excessive bleeding. In contrast, other inherited or acquired deficiencies including factor XII deficiency, ${ }^{11}$ prekallikrein deficiency, or HMWK deficiency may be clinically silent. Lupus anticoagulants can also prolong PTT without any associated bleeding problems. ${ }^{10}$

\section{CONCLUSION}

Preoperative evaluation of hemostasis is crucial to assess the risk of perioperative and postoperative bleeding. The current method of screening is to obtain a thorough history of bleeding of both the individual and the family. However, this method may not be effective for rare or asymptomatic disorders, as in this case. We suggest that routine detailed history and physical examination, along with preoperative evaluations of PTT, PT, bleeding time, and platelet count, should be considered on a case-by-case basis. The ACT performed by the anesthesiologist just prior to surgery was grossly abnormal and led to reevaluation and extensive testing. The choice of laboratory test and use of FFP should be carefully considered when evaluating anticoagulation in patients with prekallikrein deficiency. Our experience demonstrates that surgery can be safely undertaken in patients with prekallikrein deficiency.

\section{ACKNOWLEDGMENTS}

The authors have no financial or proprietary interest in the subject matter of this article.

\section{REFERENCES}

1. Hathaway WE, Belhasen LP, Hathaway HS. Evidence for a new plasma thromboplastin factor. I. Case report, coagulation studies and physicochemical properties. Blood. 1965 Nov;26 (5):521-532.

2. Girolami A, Allemand E, Bertozzi I, Candeo N, Marun S, Girolami B. Thrombotic events in patients with congenital prekallikrein deficiency: a critical evaluation of all reported cases. Acta Haematol. 2010;123(4):210-214. doi: 10.1159/000313361.

3. Meier HL, Pierce JV, Colman RW, Kaplan AP. Activation and function of human Hageman factor. The role of high molecular weight kininogen and prekallikrein. J Clin Invest. 1977 Jul;60 (1):18-31.

4. Girolami A, Scarparo P, Candeo N, Lombardi AM. Congenital prekallikrein deficiency. Expert Rev Hematol. 2010 Dec;3(6):685695. doi: 10.1586/ehm.10.69.

5. Dasanu CA, Alexandrescu DT. A case of prekallikrein deficiency resulting in severe recurrent mucosal hemorrhage. Am J Med Sci. 2009 Nov;338(5):429-430. doi: 10.1097/MAJ.0b013e3181b270bb.

6. Girolami A, Ferrari S, Cosi E, Sambado L, Girolami B. Prevalence of hypertension and its complications in congenital prekallikrein deficiency: analysis of all reported cases and clinical significance. Blood Coagul Fibrinolysis. 2015 Jul;26 (5):560-563. doi: 10.1097/MBC.0000000000000294.

7. Bojanini EU, Loaiza-Bonilla A, Pimentel A. Prekallikrein deficiency presenting as recurrent cerebrovascular accident: case report and review of the literature. Case Rep Hematol. 2012;2012:723204. doi: 10.1155/2012/723204.

8. Sugi T, Makino T. Factor XIl, kininogen and plasma prekallikrein in abnormal pregnancies. Curr Drug Targets. 2005 Aug;6(5):551557.

9. Odumosu MC, Yoong WC, Fakokunde AF. Fletcher factor deficiency in a woman requiring emergency caesarean section. J Obstet Gynaecol. 2009 Jul;29(5):442. doi: 10.1080/ 01443610902952554.

10. Oram MP, Mumford AD, Morse C, Underwood S. Management of anticoagulation for coronary artery bypass surgery in a patient with severe prekallikrein deficiency. J Cardiothorac Vasc Anesth. 2006 Aug;20(4):580-582.

11. Lämmle B, Wuillemin WA, Huber I, et al. Thromboembolism and bleeding tendency in congenital factor XII deficiency-a study on 74 subjects from 14 Swiss families. Thromb Haemost. 1991 Feb 12;65(2):117-121.

This article meets the Accreditation Council for Graduate Medical Education and the American Board of Medical Specialties Maintenance of Certification competencies for Patient Care and Medical Knowledge. 